\title{
SPIRAL GALAXIES ROTATION CURVES
}

\section{WITH A LOGARITHMIC CORRECTED NEWTONIAN GRAVITATIONAL POTENTIAL}

\author{
J.C. Fabri: 1 and J. Pereira Campos2, \\ Departamento de Física, Universidade Federal do Espírito Santo \\ 29060-900, Vitória, Espírito Santo, Brazil
}

\begin{abstract}
We analyze the rotation curves of 10 spiral galaxies with a newtonian potential corrected with an extra logarithmic term, using a disc modelization for the spiral galaxies. There is a new constant associated with the extra term in the potential. The rotation curve of the chosen sample of spiral galaxies is well reproduced for a given range of the new constant. It is argued that this correction can have its origin from string configurations. The compatibility of this correction with local physics is discussed.
\end{abstract}

Pacs numbers: 98.80.-k, 98.80.Es

The observed features of the rotation curves of gravitationally bound objects, like stars or gas clouds, in spiral galaxies is one of the most important evidence to the existence of dark matter. Spiral galaxies is composed essentially of a very dense central bulge and a flat disc containing spiral arms. At large distance from the center of the galaxy, the keplerian orbits of stars or gas clouds should exhibit velocities decreasing with the distance to the center. However, observations indicate that the velocities become constant (or even increase) at very large distances to the center of the galaxy. This feature seems to be universal, and it can not be explained by the visible matter present in the galaxy, neither by an additional amount of invisible baryonic matter (baryonic dark matter). It is largely accepted today in the astrophysics community that the only consistent explanation is the existence of a large amount of non-baryonic dark matter, which composes a huge halo around the galaxy extending for a distance many times larger than the visible radius of the galaxy. The reader is addressed to the reviews articles on the subject for the evidences for the existence of non-baryonic dark matter and for the candidates to represent it [1, 2]. For specific models concerning the dark matter hypothesis, see reference [11.

Concerning the candidates to form this "dark halo" around the spiral (and perhaps even the elliptic) galaxies, those most frequently quoted are the axion and the neutralinos. Axions are relics of "grand

\footnotetext{
1e-mail: fabris@pq.cnpq.br. Present address: Institut d'Astrophysique de Paris (IAP), 98bis Boulevard Arago, 75014 Paris, France.

${ }^{2}$ e-mail: jpcampospt@gmail.com
} 
unified theory" (GUT) phase in the primordial universe. Axions are particles with small mass, $m_{A} \sim 5 \mathrm{eV}$, but with a very small cross section, so that a gas of axions may be approximated by a pressureless, nonrelativistic, fluid in spite of its small mass. Neutralinos are the less massive, electrically neutral, stable supersymmetric particles. Both axions and neutralinos have all the characteristic one can ask for a nonbaryonic dark matter: they do not interact with light, having at the same time a very small cross section, behaving essentially as a cold (pressureless) fluid. Cosmologically, they also could play an important role in the structure formation since they would decouple very early from the radiative gas that dominates the matter content of the universe before the matter dominated phase.

In spite of its many success in describing the dynamics of galaxies, and even cluster of galaxies, the dark matter model has, for the moment, a major drawback: no one of its candidates comes from a experimentally tested theory. Axions and neutralinos, in special, remain theoretical proposals. This fact legitimates the search of alternatives to the dark matter model. One of them is to suppose that the ordinary laws of gravity are not valid from galactic scales on. This alternative is quite attractive since the proportion of dark matter, with respect to baryonic matter, required to explain the dynamics of virialized structure like galaxies and clusters of galaxies increases as the scales of the structure increases: clusters of galaxies ask for a much higher proportion than galaxies, for example [4]. A recent analysis of supercluster dynamics suggests the possibility of a new long range force [5]. Another possibility is to keep the gravity theory intact, but to modify the law of inertia, as it has been done by the MOND theory, which modifies the Newton second law [6]. This proposal has been particularly successful in reproducing the rotation curves of spiral galaxies [7. The application of MOND to clusters of galaxy is more problematic.

In this work we will opt to investigate the consequences of changing the newtonian gravity law. This will be done by adding an extra logarithmic term to the newtonian potential. This has the evident advantage of giving a constant velocity to the keplerian trajectories for large values of the radial distance $r$. But, it has also a theoretical motivation: string-like objects would contribute with a logarithmic type potential if the energy and the radial pressure are much larger than the transverse pressure. Hence a gas of cosmic string would have a different gravitational behavior compared with a gas of point-like objects. The relativistic analysis of a gas of string has been carried out in reference [8]. The connection with a logarithmic extra term in the newtonian potential has been presented in reference $[9]$. The logarithmic extra term becomes exact when the strings composing the gas are all radially aligned.

We will compute here a more detail galactic model with the extra logarithmic potential term, performing also a comparison of theoretical predictions and observational data for a given sample of spiral galaxies. Using a flat thin disc approximation for representing the spiral galaxies, we will show that the logarithmic modification leads to very good results. We remark, en passant, that a relativistic non-linear theory of the type $f(R), R$ being the Ricci scalar, leads to a modification of the newtonian potential with an extra term of the type $r^{\beta}$. The comparison for such kind of models (not including a logarithmic 
potential term) with the rotation curve of $L S B$ spiral galaxies has been performed in references [10, 11]. A preferred value $\beta \sim 1.7$ has been found [11. Here we will use a sample of $9 L S B$ galaxies described in reference [12]. To this sample, we add a galaxy from reference [11] whose rotation curve extend very far from the luminous disk.

Let us consider the modified newtonian potential as

$$
\phi(\vec{r})=-\frac{G m}{r}-\alpha G m \ln \left(\frac{r}{r_{0}}\right),
$$

where $\alpha$ is a new parameter with dimension of $L^{-1}$ (which must be negative in order to give an attractive extra term in the newtonian force) and $r_{0}$ is an arbitrary constant. We will treat the new (dimensional) constant $\alpha$ as arbitrary, even if it must have a connection with the string tension. Considering a smooth distribution of mass, the potential at a position $\vec{r}$ is given by

$$
\phi(\vec{r})=-G \int_{V^{\prime}} \frac{\rho\left(\vec{r}^{\prime}\right)}{\left|\vec{r}-\vec{r}^{\prime}\right|} d \vec{r}^{\prime}-\alpha G \int_{V^{\prime}} \rho\left(\vec{r}^{\prime}\right) \ln \left(\frac{\left|\vec{r}-\vec{r}^{\prime}\right|}{r_{0}}\right) d \vec{r}^{\prime}
$$

Let us consider now the approximation that a spiral galaxy is a flat disc of a given radius. In this first approach, the bulge and the spiral arms will be neglected. Hence, we must solve the integrals above in cylindrical coordinates. The disc is characterized by a surface density. We will use the exponential model for the disc [13, and we will suppose that light traces mass. Hence, the surface density is given by

$$
\Sigma(r)=\Sigma_{0} e^{-\frac{r}{r_{d}}}
$$

where $r_{d}$ is the luminosity scale of the disc, indicating how surface luminosity decreases with the distance to the center of the disc, and $r$ is the distance of the center of the disc to a given point on it.

For the pure newtonian potential, this calculation is present in some detail in reference [14. Adding the calculation to the extra term, which is quite straightforward, we find

$$
v_{c}^{2}=\frac{\pi G \Sigma_{0} r^{2}}{r_{d}}\left[I_{0}\left(\frac{r}{2 r_{d}}\right) K_{0}\left(\frac{r}{2 r_{d}}\right)-I_{1}\left(\frac{r}{2 r_{d}}\right) K_{1}\left(\frac{r}{2 r_{d}}\right)\right]+2 \pi \alpha G \Sigma_{0} r_{d}\left[e^{-\frac{r}{r_{d}}}\left(r+r_{d}\right)-r_{d}\right],
$$

where $I_{\nu}$ and $K_{\nu}$ are the modified Bessel's functions.

The first term in (4) represents the contribution of the newtonian potential, while the second one is the contribution due to the extra logarithmic term. In relation (4) there are essentially two free parameters: the central surface density $\Sigma_{0}$ and $\alpha$. We will use a sample of nine low surface brightness galaxies $(L S B)$, that is, galaxies that ask for a large amount of dark matter, to test this model.

The comparison between the observational data and the theoretical results is given through the $\chi^{2}$ statistics:

$$
\chi^{2}=\sum_{i} \frac{\left(v_{i}^{o}-v_{i}^{t}\right)^{2}}{\sigma_{i}^{2}}
$$

where $v_{i}^{o}$ is the observed velocity of the $i t h$ object, $v_{i}^{t}$ the theoretical result for this same object, and $\sigma_{i}$ the observational error bar. From this, we obtain the probability distribution

$$
P\left(\alpha, \Sigma_{0}\right)=A e^{-\chi^{2}\left(\alpha, \Sigma_{0}\right) / 2},
$$



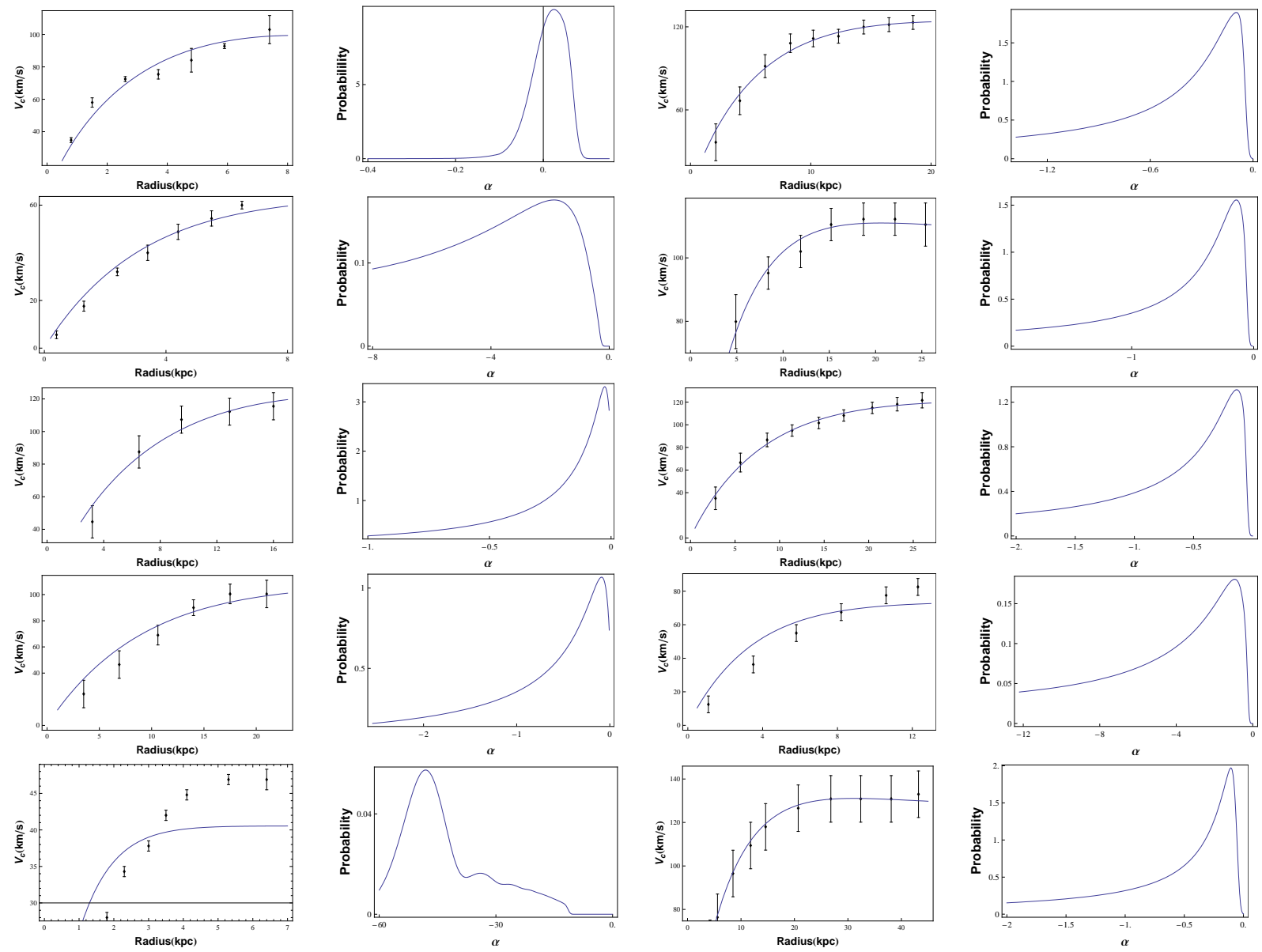

Figure 1: The rotation curve for the $L S B$ spiral galaxies $N G C 247, F 568 . v 1, N G C 3109, F 563-1, F 568-1, F 568-3$, $F 574-1, F 583-1, D D O 154$ and $U G C 128$. The continuous curve is the theoretical results using the modified newtonian potential. In the right of each rotation curve it is displayed the corresponding marginalized probability distribution for the parameter $\alpha$.

$A$ being a normalization constant. The prediction for a given parameter is obtained by integrating (marginalizing) on the other parameter, resulting in one-dimensional probability distribution. More details on this procedure can be found in reference [15, 16]. 


\begin{tabular}{|c|c|c|}
\hline Galaxy & $\alpha(2 \sigma)$ & $r_{d}$ \\
\hline NGC247 & $-0.0243_{-0.1062}^{+0.0469} k p c^{-1}$ & $2.30 k p c$ \\
\hline F568.v1 & $-0.0975_{-1.1293}^{+0.0911} k p c^{-1}$ & $4.86 k p c$ \\
\hline NGC3109 & $-1.8643_{-5.8406}^{+1.1910} k p c^{-1}$ & $2.30 k p c$ \\
\hline F563-1 & $-0.1421_{-1.5390}^{+0.1181} k p c^{-1}$ & $4.29 k p c$ \\
\hline F568-1 & $-0.0261_{-1.2871}^{+0.0179} k p c^{-1}$ & $8.20 k p c$ \\
\hline F568-3 & $-0.1353_{-1.3127}^{+0.1304} k p c^{-1}$ & $6.15 k p c$ \\
\hline F574-1 & $-0.0883_{-2.1433}^{+0.0866} k p c^{-1}$ & $6.58 k p c$ \\
\hline F583-1 & $-0.9462_{-10.0875}^{+0.6937} k p c^{-1}$ & $2.43 k p c$ \\
\hline DDO154 & $-48.0835_{-3.8165}^{+15.985} k p c^{-1}$ & $0.72 k p c$ \\
\hline UGC128 & $-0.1065_{-1.6054}^{+0.0795} k p c^{-1}$ & $6.4 k p c$ \\
\hline
\end{tabular}

In the sample of 10 galaxies, two of them are dwarf and one is a barred irregular. From figure (1) and from the corresponding table, we can verify that the results are very good, giving a consistent value for the parameter $\alpha$ for the "normal" $L S B$ spiral galaxies. For the two dwarfs galaxies the results give either a very small value to $\alpha(N G C 247)$ or a too large value (NGC3109). Moreover, the results are clearly inconsistent for the irregular barred galaxy DDO154. These three cases suffer, in fact, from the simplicity of the model: these galaxies, and mainly the DDO154, are poorly approximated by a flat disc. For the other six, however, the approximation works remarkably well. For these six galaxies, the predicted value of $\alpha$ is always negative (at $2 \sigma$ confidence level), indicating an attractive extra term. There is a "concordance" value around $\alpha \sim-0.1 \mathrm{kpc}^{-1}$.

A correction of the newtonian potential with $\alpha \sim-0.1 k p c^{-1}$ may lead to the "good" rotation curves for spiral galaxies. At the same time, it does not imply a detectable effect at level of the solar system. In fact, the ratio between the normal newtonian force $F_{N}$ and the correction to this force $F_{c}$ is of the order of

$$
\frac{F_{c}}{F_{N}}=-\alpha r
$$

For a distance of some solar units, and using the indicated value for $\alpha$, this ratio is of the order $10^{-13}$. The extra acceleration it provokes in the solar system, at a distance of some solar units from the sun, is of the order $10^{-12}$ : the Pioneer anomaly is surely not due to this correction. This is reasonable, since a gas of cosmic strings should not have detectable effects at the solar system scales, otherwise cosmic stings should already been observed in local experiences.

The most important point to be stressed is the fact that the value of $\alpha$ is compatible for the group of galaxies for which the disc approximation is at least reasonable. For the dwarf galaxies this approximation is quite crude. For the irregular barred galaxy DDO154, the approximation is simply invalid. Hence, even the fact that the results for this last case is inconsistent with the other cases seems to be a positive 
aspect of the model exploited here, due to the simplicity of the modelization made. Moreover, we remark the excellent agreement with the galaxy $U G C 128$ whose observed rotation curve extend very far from the luminous disk.

\section{Acknowledgements:}

We thank CNPq (Brazil) and the french/brazilian scientific cooperation CAPES/COFECUB (project number 506/05) for partial financial support. We thank also Winfried Zimdahl for reading the text and his suggestions.

\section{References}

[1] L. Bergström, Rep. Prog. Phys. 63, 793 (2000).

[2] G. Bertone, D. Hooper and J. Silk, Phys. Rep. 405, 279 (2005).

[3] P. Salucci and A. Borriello, Lec. Not. Phys. 616, 66 (2003).

[4] A. Kashlinsky, Phys. Rep. 307, 67 (1998).

[5] G.R. Farrar and R.A. Rosen, A new force in the dark sector?, astro-ph/0610298

[6] M. Milgrom, Astrophys. J. bf 270, 365 (1983).

[7] R.H. Sanders, Modified gravity without dark matter, astro-ph/0601431.

[8] P. Letelier, Phys. Rev. D20, 1294 (1979).

[9] H.H. Soleng, 27, 4 (1995).

[10] S. Capozziello, V.F. Cardone and A. Troisi, Month. Not. R. Astron. Soc. 375, 1423 (2007).

[11] C.F. Martins and P. Salucci, Analysis of rotation curves in the framework of $R^{n}$ gravity, astro-ph/0703243.

[12] F.C. van den Bosch, B.E. Robertson, J.J. Dalcanton and W.J.G. de Blok, Astron. J. 119, 1579 (2000).

[13] K.C. Freeman, Astrophys. J. 160, 811 (1970).

[14] J. Binney and S. Tremaine, Galactic dynamics, Princeton University Press, Princeton (1987).

[15] R. Colistete Jr. and J.C. Fabris, Class. Quant. Grav. 22, 2813 (2005).

[16] R. Colistete Jr., J.C. Fabris and S.V.B. Gonçalves, Int. J. Mod. Phys. D14, 775 (2005). 